PHYSICAL REVIEW D 96, 049902(E) (2017)

\title{
Publisher's Note: Role of symmetries in the Kerr-Schild derivation of the Kerr black hole \\ [Phys. Rev. D 94, 064073 (2016)]
}

Eloy Ayon-Beato, Mokhtar Hassaïne, and Daniel Higuita-Borja

(Received 25 July 2017; published 7 August 2017)

DOI: 10.1103/PhysRevD.96.049902

This paper was published online on 26 September 2016 with incorrect author affiliations. The author affiliations should read as

Eloy Ayón-Beato ${ }^{1,2, *}$ Mokhtar Hassaïne, ${ }^{3, \dagger}$ and Daniel Higuita-Borja ${ }^{1,2, \ddagger}$

${ }^{1}$ Departamento de Física, CINVESTAV-IPN, Apartado Postal 14-740, 07000 México Distrito Federal, México

${ }^{2}$ Instituto de Ciencias Físicas y Matemáticas, Universidad Austral de Chile, Casilla 567 Valdivia, Chile.

${ }^{3}$ Instituto de Matemática y Física, Universidad de Talca, Casilla 747, Talca, Chile

The author affiliations have been corrected as of 28 July 2017. The author affiliations are incorrect in the printed version of the journal. 